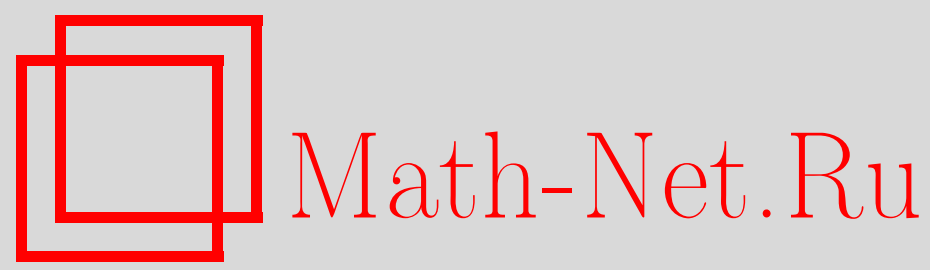

Б. В. Заятуев, Об одном классе почти эрмитовой структуры на касательном расслоении, Матем. заметки, 2004, том 76, выпуск 5, 732-739

DOI: https://doi.org/10.4213/mzm148

Использование Общероссийского математического портала Math-Net.Ru подразумевает, что вы прочитали и согласны с пользовательским соглашением http://www.mathnet.ru/rus/agreement

Параметры загрузки:

IP: 54.174 .149 .18

26 апреля 2023 г., 02:30:53

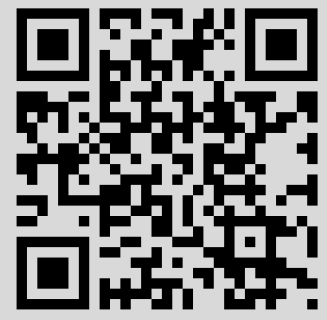




\title{
ОБ ОДНОМ КЛАССЕ ПОЧТИ ЭРМИТОВОЙ СТРУКТУРЫ НА КАСАТЕЛЬНОМ РАССЛОЕНИИ
}

Б.В. Заятуев

\begin{abstract}
На касательном расслоении построена новая почти эрмитова структура, принадлежащая к антиинвариантному типу. Получены критерии принадлежности этой почти эрмитовой структуры ко всем классам Грея-Хервеллы. В частности, доказано, что касательные расслоения над келеровым и семикелеровым многообразиями имеют соответственно структуру почти келерова и семикелерова многообразия.

Библиографория: 18 названий.
\end{abstract}

Касательные расслоения особенно богаты различньми дифференциально-геометрическими структурами, которые тем или иным образом индуцируются на нем из геометрических структур его базы. Еще в 1962 году, одновременно, Домбровский [1] и Татибана, Окумура [2], используя понятия горизонтального и вертикального лифтов, показали, что на касательном расслоении над римановым многообразием внутренним образом определена почти келерова структура. Другие новые, полученные за последнее время, примеры почти эрмитовых структур на касательном расслоении можно найти, например, в работах [3]-[5]. В настояшей работе, в отличие от большинства других, мы рассматриваем касательное расслоение над почти эрмитовым многообразием. Показано, что на нем естественным образом индуцируется почти эрмитова структура антиинвариантного типа и получены условия принадлежности этой структуры ко всем классам Грея-Хервеллы [6]. Основные результаты работы получены вычислением в формализме Кошуля и методом структурных уравнений почти эрмитовой структуры на пространстве расслоения $A$-реперов [7].

Пусть $(M, J, g)$ - почти эрмитово многообразие. Определим на касательном расслоении $T(M)$ линейньй оператор $\widetilde{J}$, действуюший в $C^{\infty}(T M)$-модуле $\mathfrak{X}(T M)$ гладких векторных полей на $T(M)$ следующим образом:

$$
\widetilde{J}\left(X^{H}\right)=(J X)^{V}, \quad \widetilde{J}\left(X^{V}\right)=(J X)^{H},
$$

где $(\ldots)^{H},(\ldots)^{V}$ - горизонтальные и вертикальные лифты [8]. Очевидно, что $\widetilde{J}$ является почти комплексной структурой на $T(M)$, обладающей следующим свойством:

$$
\widetilde{J}(\mathscr{H}) \subset \mathscr{V}
$$

где $\mathscr{H}$ - горизонтальное, $\mathscr{V}$ - вертикальное распределения на $T(M)$. 
В [9] (см., также, [10], [11]) на касательном расслоении $T(M)$ была рассмотрена почти эрмитова структура инвариантного типа (иначе, 1-го рода) с римановой метрикой $\widetilde{g}$, определенной следующим образом:

$$
\widetilde{g}\left(X^{H}, Y^{H}\right)=\lambda g(X, Y), \quad \widetilde{g}\left(X^{H}, Y^{V}\right)=0, \quad \widetilde{g}\left(X^{V}, Y^{V}\right)=g(X, Y),
$$

где $\lambda>0, \lambda \in C^{\infty}(M)$. Причем относительно римановой связности $\widetilde{\nabla}$ метрики $\widetilde{g}$ имеют место следующие формулы:

$$
\begin{aligned}
& \widetilde{\nabla}_{X^{H}} Y^{H}=\left(\dot{\nabla}_{X} Y\right)^{V}-\frac{1}{2}(R(X, Y) y)^{V}, \\
& \widetilde{\nabla}_{X^{H}} Y^{V}=\frac{1}{2 \lambda}(R(y, Y) X)^{H}+\left(\nabla_{X} Y\right)^{V}, \\
& \widetilde{\nabla}_{X^{V}} Y^{H}=\frac{1}{2 \lambda}(R(y, X) Y)^{H}, \\
& \widetilde{\nabla}_{X^{V}} Y^{V}=0,
\end{aligned}
$$

где $\nabla, \dot{\nabla}-$ соответственно римановы связности метрик $g$ и $\lambda g ; R$ - тензор кривизны связности $\nabla$, а $R(y, X) Y$, где $y=\left(y^{1}, \ldots, y^{n}\right)$ - слоевые координаты, рассматривается как элемент $C^{\infty}(T(M))$-модуля $C^{\infty}(T(M)) \otimes \mathfrak{X}(M)$ (см. [3]).

Непосредственной проверкой можно убедиться, что пара $\{\widetilde{J}, \widetilde{g}\}$ образует почти эрмитову структуру на $T(M)$ только в том случае, когда $\lambda=1$, т.е. когда $\widetilde{g}$-метрика Сасаки. Поэтому в дальнейшем через $\widetilde{g}$ мы будем обозначать именно эту метрику. Почти эрмитову структуру $\{\widetilde{J}, \widetilde{g}\}$ мы назовем антиинвариантной структурой (или структурой 2-го рода).

Вычислив с учетом (1) (при $\lambda=1$ ) ковариантную производную

$$
\widetilde{\nabla}_{\bar{X}}(\widetilde{J}) \bar{Y}=\widetilde{\nabla}_{\bar{X}}(\widetilde{J} \bar{Y})-\widetilde{J} \widetilde{\nabla}_{\bar{X}} \bar{Y}
$$

структурного оператора $\widetilde{J}$, получим

$$
\begin{aligned}
& \widetilde{\nabla}_{X^{H}}(\widetilde{J}) Y^{H}=\frac{1}{2}(R(y, J Y) X+J R(X, Y) y)^{H}+\left(\nabla_{X}(J) Y\right)^{V}, \\
& \widetilde{\nabla}_{X^{H}}(\widetilde{J}) Y^{V}=\left(\nabla_{X}(J) Y\right)^{H}-\frac{1}{2}(R(X, J Y) y+J R(y, Y) X)^{V}, \\
& \widetilde{\nabla}_{X^{V}}(\widetilde{J}) Y^{H}=-\frac{1}{2}(J R(y, X) Y)^{V}, \\
& \widetilde{\nabla}_{X^{V}}(\widetilde{J}) Y^{V}=\frac{1}{2}(R(y, X) J Y)^{H} .
\end{aligned}
$$

В 1980 году А. Грей и Л. Хервелла, исходя из линейного представления унитарной группы $U(m)$ на одном специальном линейном пространстве $W$, выделили, в известном смысле, полную систему почти эрмитовых структур. Оказалось, что действие $U(m)$ на $W$ вполне приводимо и $W$ разлагается в прямую сумму четырех неприводимых компонент $W_{1}, W_{2}, W_{3}, W_{4}(m>2)$, которые определяют 16 классов почти эрмитовых структур на гладком многообразии $\left(M^{2 m}, J, g\right)$. 
ОПРЕДЕЛЕНИЕ 1. Почти эрмитово многообразие $(M, J, g)$ называется келеровы.м, если структурньй оператор $J$ параллелен, т.е. $\nabla J=0$.

Из условия параллельности почти комплексной структуры $\widetilde{J}$ относительно римановой связности $\widetilde{\nabla}$ с учетом соотношений $(2)$ имеем, в частности,

$$
\begin{aligned}
\left(\nabla_{X}(J) Y\right)^{H}- & \frac{1}{2}(R(X, J Y) y+J R(y, Y) X)^{V}=0 \\
& (R(y, X) J Y)^{H}=0 .
\end{aligned}
$$

Отсюда, очевидно, что $\nabla J=0$ и $R=0$. Следовательно, справедливо

ПРЕДЛОЖЕНИЕ 1. Касательное расслоение $\{T(M), \widetilde{J}, \widetilde{g}\}-$ келерово многообразие тогда и только тогда, когда $(M, J, g)$ - плоское келерово многообразие.

ОПРЕДЕЛЕНИЕ $2[6]$. Почти эрмитово многообразие $(M, J, g)$ называется $G_{1}$-многообразием Видаля-Хервелль, если

$$
\nabla_{X}(\Omega)(X, Y)-\nabla_{J X}(\Omega)(J X, Y)=0, \quad X, Y \in \mathfrak{X}(M)
$$

где $\Omega(X, Y)=g(J X, Y)-$ фундаментальная форма.

Отметим, что $G_{1}$-многообразия относятся к классу $W_{1} \oplus W_{3} \oplus W_{4}$ в классификации Грея-Хервеллы. Возможно, впервые они были введены в работах Накаямы [12], а затем более подробно изучены Видалем и Хервеллой [13].

Используя поляризацию, запишем определяющее условие (3) относительно структуры $\{\widetilde{J}, \widetilde{g}\}$ в следующем эквивалентном виде:

$$
\widetilde{\nabla}_{\bar{X}}(\widetilde{J}) \bar{Y}-\widetilde{\nabla}_{\tilde{J} \bar{X}}(\widetilde{J}) \widetilde{J} \bar{Y}+\widetilde{\nabla}_{\bar{Y}}(\bar{X})-\widetilde{\nabla}_{\tilde{J} \bar{Y}}(\widetilde{J}) \widetilde{J} \bar{X}=0
$$

где $\bar{X}, \bar{Y} \in \mathfrak{X}(T(M))$. Тогда с учетом формул (2) имеем

1) при $\bar{X}=X^{H}, \bar{Y}=Y^{H}$

$$
\frac{1}{2}(R(y, J Y) X+R(y, J X) Y)^{H}+\left(\nabla_{X}(J) Y+\nabla_{Y}(J) X\right)^{V}=0
$$

2) при $\bar{X}=X^{H}, \bar{Y}=Y^{V}$

$$
\frac{1}{2}(-J R(y, J Y) J X+J R(y, J X) J Y)^{H}+\left(\nabla_{X}(J) Y-\nabla_{J Y}(J) J X\right)^{V}=0
$$

3) при $\bar{X}=X^{V}, \bar{Y}=Y^{V}$ получим соотношение, эквивалентное 1).

Отсюда следует, что соотношение (4) эквивалентно двум условиям (которые имеют место на базе касательного расслоения)

$$
\begin{gathered}
\nabla_{X}(J) Y+\nabla_{Y}(J) X=0, \\
R(y, J Y) X+R(y, J X) Y=0,
\end{gathered}
$$


где $X, Y \in \mathfrak{X}(M)$. Как известно [14], соотношение (5) является определяющим условием приближенно келеровых многообразий. Приближенно келеровы многообразия представляют собой один из наиболее глубоко изученных и интересных классов почти эрмитовых структур (см., например, [14], [7], [15]). Далее, напомним, что на пространстве расслоения $A$-реперов [16] тензоры $\{J, g\}$ имеют следуюшие компоненты:

$$
\begin{gathered}
J_{b}^{a}=i \delta_{b}^{a}, \quad J_{\widehat{b}}^{\widehat{a}}=-i \delta_{b}^{a}, \quad J_{b}^{\widehat{a}}=J_{\widehat{b}}^{a}=0, \\
g_{a \widehat{b}}=\delta_{b}^{a}, \quad g_{a b}=g_{\widehat{a} \widehat{b}}=0,
\end{gathered}
$$

где $a=1, \ldots, m, \widehat{a}=a+m, \operatorname{dim} M=2 m$. Записав соотношение (6) на пространстве расслоения $A$-реперов, получим, что оно эквивалентно условиям

$$
\begin{aligned}
& \text { 1) } R_{a b c d}=0 ; \\
& \text { 2) } R_{\widehat{a} b c \hat{d}}=0 ; \\
& \text { 3) } R_{a b \widehat{c} \widehat{d}}=0 .
\end{aligned}
$$

Как известно [7], существенные (с точностью до комплексного сопряжения) элементы спектра тензора кривизны приближенно келеровых многообразий на пространстве расслоения $A$-реперов имеют вид

$$
\begin{aligned}
& \text { 1) } R_{a b \widehat{c} \widehat{d}}=-2 B_{a b h} B^{h c d} \\
& \text { 2) } R_{a \widehat{b} \widehat{c} d}=B_{a d h} B^{h b c}+A_{a d}^{b c} \\
& \text { 3) } R_{a b c d}=-2 B_{a b[c d]} \\
& \text { 4) } R_{a \widehat{b} c d}=0
\end{aligned}
$$

где $\left\{B^{h c d}\right\},\left\{B_{a b h}\right\}$ - соответственно компоненты структурных тензоров 1-го и 2-го рода. В случае приближенно келеровых многообразий они кососимметричны по всем индексам и их обращение в нуль равносильно келеровости структуры $\{J, g\} .\left\{B^{a b c d}\right\}-$ элементы ковариантного дифференциала в почти эрмитовой связности [16] тензора $\left\{B^{a b c}\right\} ;\left\{A_{c d}^{a b}\right\}$ - компоненты тензора голоморфной секционной кривизны.

Из $\left(7_{3}\right)$ с учетом $\left(8_{1}\right)$ имеем

$$
B_{a b h} B^{h c d}=0 .
$$

Свертывая это соотношение соответственно по индексам $(a, c),(b, d)$ с учетом свойств симметрии и комплексной сопряженности соответствующих компонент структурных тензоров, получим

$$
B_{a b h} B^{h a b}=\sum_{h} \bar{B}^{h a b} B^{h a b}=\sum_{h}\left|B^{h a b}\right|^{2}=0 .
$$

Отсюда следует

$$
B_{a b c}=B^{a b c}=0 .
$$

Таким образом, $(M, J, g)$ - келерово многообразие. Из условия келеровости также следует, что $B^{a b c d}=0$. Кроме того, из соотношений $\left(7_{2}\right)$ и $\left(8_{2}\right)$ получаем

$$
A_{c d}^{a b}=0 .
$$

Следовательно, $R=0$, т.е. $(M, g)$ - плоское многообразие. Обратимость полученных утверждений очевидна; тем самым, доказано 
ПРЕДЛОЖЕНИЕ 2. Касательное расслоение $\{T(M), \widetilde{J}, \widetilde{g}\}-G_{1}$-многообразие тогда и только тогда, когда $(M, J, g)$ - плоское келерово многообразие.

Как показано в [6], имеет место следующее включение классов:

$$
\{0\} \subset W_{i} \subset W_{i} \oplus W_{j} \subset W_{i} \oplus W_{j} \oplus W_{k},
$$

где $i, j, k,=1,2,3,4$. С учетом этого и предложений 1,2 получаем

СлЕДСТВИЕ 1. Относительно структуры $\{\widetilde{J}, \widetilde{g}\}$ имеет место

$$
\{0\}=W_{1}=W_{3}=W_{4}=W_{1} \oplus W_{3}=W_{1} \oplus W_{4}=W_{3} \oplus W_{4}=W_{1} \oplus W_{3} \oplus W_{4} .
$$

ОПРЕДЕЛЕНИЕ 3. Почти эрмитово многообразие $(M, J, g)$ называется почтu келеровым, если его фундаментальная форма замкнута, т.е. $d \Omega=0$.

Напомним, что почти келеровы многообразия представляют класс $W_{2}$. Они занимают видное место среди почти эрмитовых многообразий в связи с известной связью с симплектическими многообразиями, играющими фундаментальную роль в аналитической механике.

Используя кососимметричность фундаментальной формы $\widetilde{\Omega}$ и отсутствие кручения у римановой связности $\widetilde{\nabla}$, можем записать условие почти келеровости структуры $\{\widetilde{J}, \widetilde{g}\}$ в следующем виде:

$$
\widetilde{g}\left(\widetilde{\nabla}_{\bar{X}}(\widetilde{J}) \bar{Y}, \bar{Z}\right)+\widetilde{g}(\widetilde{\nabla} \bar{Z}(\widetilde{J}) \bar{X}, \bar{Y})+\widetilde{g}\left(\widetilde{\nabla}_{\bar{Y}}(\widetilde{J}) \bar{Z}, \bar{X}\right)=0
$$

где $\bar{X}, \bar{Y}, \bar{Z} \in \mathfrak{X}(T(M))$. Тогда с учетом формул (2) имеем

1) при $\bar{X}=X^{H}, \bar{Y}=Y^{H}, \bar{Z}=Z^{H}$

$$
R(Z, X, y, J Y)+R(X, Y, y, J Z)+R(Y, Z, y, J X)=0
$$

2) при $\bar{X}=X^{H}, \bar{Y}=Y^{H}, \bar{Z}=Z^{V}$

$$
g\left(\nabla_{X}(J) Y, Z\right)+g\left(\nabla_{Y}(J) Z, X\right)=0 ;
$$

3) в оставшихся возможных вариантах получим тождество $0=0$.

Из $\left(10_{2}\right)$, используя циклическую перестановку аргументов, получим

$$
g\left(\nabla_{Y}(J) Z, X\right)=0
$$

Следовательно, $\nabla J=0$, т.е. $(M, J, g)$ - келерово многообразие. Непосредственной проверкой можно убедиться, что в силу известных тождеств кривизны келеровых многообразий и тождества Риччи равенство $\left(10_{1}\right)$ вьполняется тождественно. Таким образом, справедливо

ПРЕДЛОЖЕНИЕ 3. Касательное расслоение $\{T(M), \widetilde{J}, \widetilde{g}\}-$ почти келерово многообразие тогда и только тогда, когда $(M, J, g)$ - келерово многообразие. 
ОПРЕДЕЛЕНИЕ 4. Почти эрмитово многообразие $(M, J, g)$ называется $G_{2}$-многообразием Видаля-Хервелль, если

$$
\sigma_{X Y Z}\left(\nabla_{X}(\Omega)(X, Z)-\nabla_{J X}(\Omega)(J X, Z)\right)=0,
$$

$X, Y, Z \in \mathfrak{X}(M)$, где $\sigma_{X Y Z}$ обозначает циклическое суммирование.

Напомним, что $G_{2}$-многообразия относятся к классу $W_{2} \oplus W_{3} \oplus W_{4}$.

Запишем определяющее условие (11) относительно структуры $\{\widetilde{J}, \widetilde{g}\}$ в следующем виде:

$$
\sigma_{\overline{X Y Z}}\left\{\widetilde{g}\left(\widetilde{\nabla}_{\bar{X}}(\widetilde{J}) \bar{Y}, \bar{Z}\right)-\widetilde{g}\left(\widetilde{\nabla}_{\tilde{J}}(\widetilde{J}) \widetilde{J} \bar{Y}, \bar{Z}\right)\right\}=0,
$$

где $\bar{X}, \bar{Y}, \bar{Z} \in \mathfrak{X}(T(M))$. Положив в (12) соответственно $\bar{X}=X^{H}, \bar{Y}=Y^{H}, \bar{Z}=Z^{H}$ и $\bar{X}=X^{H}, \bar{Y}=Y^{H}, \bar{Z}=Z^{V}$ (остальные возможные варианты дадут только тождества) с учетом формул (2) получим

$$
\begin{array}{ll}
\text { 1) } & R(X, Y, y, J Z)+R(Y, Z, y, J X)+R(Z, X, y, J Y)=0 \\
\text { 2) } & R(y, Z, X, J Y)+R(Y, y, X, J Z)+R(J X, y, J Y, J Z)=0 \\
\text { 3) } & g\left(\nabla_{X}(J) Y, Z\right)+g\left(\nabla_{Y}(J) Z, X\right)-g\left(\nabla_{J Z}(J) J X, Y\right)=0 \\
\text { 4) } & g\left(\nabla_{J X}(J) J Y, Z\right)+g\left(\nabla_{J Y}(J) J Z, X\right)+g\left(\nabla_{J Z}(J) J X, Y\right)=0 .
\end{array}
$$

Из $\left(13_{3}\right)$ с учетом $\left(13_{4}\right)$ имеем

$$
g\left(\nabla_{X}(J) Y, Z\right)+g\left(\nabla_{Y}(J) Z, X\right)=-g\left(\nabla_{J X}(J) J Y, Z\right)-g\left(\nabla_{J Y}(J) J Z, X\right) .
$$

Отсюда, использовав циклическую перестановку, получим

$$
g\left(\nabla_{Y}(J) Z, X\right)=-g\left(\nabla_{J Y}(J) J Z, X\right) .
$$

Следовательно,

$$
\nabla_{Y}(J) Z+\nabla_{J Y}(J) J Z=0 .
$$

С учетом этого соотношения $\left(13_{3}\right)$ примет вид

$$
g\left(\nabla_{X}(J) Y, Z\right)+g\left(\nabla_{Y}(J) Z, X\right)+g\left(\nabla_{Z}(J) X, Y\right)=0,
$$

т.е. $d \Omega=0$. Таким образом, $(M, J, g)$ - почти келерово многообразие. Далее, записав соотношения $\left(13_{1}\right)$ и $\left(13_{2}\right)$ на пространстве расслоения $A$-реперов, получим, что они эквивалентны условиям

$$
\begin{aligned}
& \text { 1) } R_{a b \widehat{c} \widehat{d}}=0 ; \\
& \text { 2) } R_{\widehat{a} b c d}=0 ; \\
& \text { 3) } R_{a b c d}=0 .
\end{aligned}
$$

Как известно [17], соответствующие элементы спектра тензора кривизны почти келеровых многообразий имеют вид
1) $R_{a b \widehat{c} \widehat{d}}=-4 B^{h c d} B_{a b h}$;
2) $R_{\widehat{a} b c d}=B_{b c d}{ }^{a}$;
3) $R_{a b c d}=-4 B_{[c|a b| d]}$; 
где структурные тензоры 1-го и 2-го рода удовлетворяют соответственно условиям $B^{[a b c]}=0$ и $B_{[a b c]}=0 ;\left\{B_{a b c d}, B_{a b c}{ }^{d}\right\}-$ компоненты ковариантной производной в почти эрмитовой связности структурного тензора $\left\{B_{a b c}\right\}$. Из $\left(14_{1}\right)$ и $\left(15_{1}\right)$ имеем

$$
B^{h a b} B_{h c d}=0 \text {. }
$$

Свернув это выражение соответственно по индексам $(a, c)$ и $(b, d)$, получим

$$
B^{a b c}=B_{a b c}=0 .
$$

И как следствие

$$
B_{a b c d}=B_{a b c}{ }^{d}=0 .
$$

Следовательно, $(M, J, g)$ - келерово многообразие. Кроме того, очевидно, что соотношения (14) вьполняются теперь тождественно. Таким образом, справедливо

ПРЕДЛОЖЕНИЕ 4. Касательное расслоение $\{T(M), \widetilde{J}, \widetilde{g}\}-G_{2}$-многообразие тогда и только тогда, когда $(M, J, g)$ - келерово многообразие.

Непосредственная проверка также показьвает, что класс $W_{1} \oplus W_{2} \oplus W_{4}=W_{2}$. Из этого и предложений 3 и 4 получаем

СлЕДСТВИЕ 2. Относительно структуры $\{\widetilde{J}, \widetilde{g}\}$ имеет место

$$
W_{2}=W_{1} \oplus W_{2}=W_{2} \oplus W_{3}=W_{2} \oplus W_{4}=W_{1} \oplus W_{2} \oplus W_{4}=W_{2} \oplus W_{3} \oplus W_{4} .
$$

ОПРЕДЕЛЕНИЕ 5. Почти эрмитово многообразие $(M, J, g)$ назьвается семикелеро$\boldsymbol{в b . M , ~ е с л и ~ е г о ~ ф у н д а м е н т а л ь н а я ~ ф о р м а ~ к о з а м к н у т а , ~ т . е . ~} \delta \Omega=0$.

Семикелеровы многообразия относятся к классу $W_{1} \oplus W_{2} \oplus W_{3}$. Эти многообразия были впервые введены Апте [18] и изучались Татибаной, Греем и др. Они являются обобщением почти келеровых и квазикелеровых многообразий.

Введем в рассмотрение $\left\{e_{i}\right\}=\left\{e_{a}, J e_{a}\right\}$ - вешественно адаптированньй (локальный) базис на многообразии $M$ и положим $\left\{\widetilde{e}_{i}\right\}=\left\{\left(e_{a}\right)^{H},\left(J e_{a}\right)^{H}\right\}$, где $i=1, \ldots, n$, $a=1, \ldots, n / 2=m$. Тогда

$$
\delta \widetilde{\Omega}(\bar{X})=\sum_{i=1}^{n}\left\{\widetilde{g}\left(\widetilde{\nabla}_{\widetilde{e}_{i}}(\widetilde{J}) \widetilde{e}_{i}, \bar{X}\right)+\widetilde{g}\left(\widetilde{\nabla}{\widetilde{J} \widetilde{e}_{i}}_{i}(\widetilde{J}) \widetilde{J} \widetilde{e}_{i}, \bar{X}\right)\right\} .
$$

С учетом формул (2) имеем

$$
\begin{aligned}
& \delta \widetilde{\Omega}\left(X^{H}\right)=\frac{1}{2} \sum_{i=1}^{n}\left\{R\left(X, e_{i}, y, J e_{i}\right)-R\left(X, e_{i}, y, J e_{i}\right)\right\}=0, \\
& \delta \widetilde{\Omega}\left(X^{V}\right)=\sum_{i=1}^{n} g\left(\nabla_{i}(J) e_{i}, X\right)=\delta \Omega(X) .
\end{aligned}
$$

Отсюда следует, что справедливо

ПРЕДЛОЖЕНИЕ 5. Касательное расслоение $\{T(M), \widetilde{J}, \widetilde{g}\}$ семикелерово тогда $и$ только тогда, когда $(M, J, g)$ - семикелерово многообразие.

Из следствий 1,2 получаем

СлЕдСтвИЕ 3. Касательные расслоения $T(M)$ над приближенно келеровыми, почти келеровыми и квазикелеровыми многообразиями $(M, J, g)$ имеют каноническую структуру семикелерова многообразия относительно $\{\widetilde{J}, \widetilde{g}\}$. 


\section{СПИСОК ЦИТИРОВАННОЙ ЛИТЕРАТУРЫ}

[1] Dombrovski P. On the geometry of the tangent bundles // J. Reine Angew. Math. 1962. V. 210. P. 73-88.

[2] Tachibana Shun-ichi, Okumura M. On the almost-complex structure of tangent bundles of Riemannian spaces // Tôhoku Math. J. (2). 1962. V. 14. P. 156-161.

[3] Кириченко В.Ф., Залтуев Б. В.Дифференциальная геометрия тангенциальных эрмитовых поверхностей // УМН. 1996. №4. С. 209-210.

[4] Tahare M., Watanabe V. Natural almost Hermition, Hermition and Kaehlerian metrics on the tangent bundles // Math. J. Toyama Univ. 1997. V. 20. P. 35-41.

[5] Papaghiuc N. The new example Kaehlerian structure on the tangent bundele of space form // Demonstr. Math. 1998. V. 31. № 4. P. 81-87.

[6] Grey A., Hervella L. M. The sixteen classes of almost Hermitian manifolds and their linear ivariants // Ann. Math. Pure Appl. 1980. V. 123. № 4. P. 35-58.

[7] Кириченко В.Ф. $K$-пространства постоянной голомоморфной секционной кривизны // Матем. заметки. 1976. Т. 19. № 5. С. 803-814.

[8] Yano K., Ishihara S. Tangent and Cotangent Bundles. New York: Marcel Dekker, 1973.

[9] Zayatuev B. V. On geometry of tangent Hermition surfaces // Webs and Quasigroups. T. S. U. 1995. P. 139-143.

[10] Заятуев Б.В. О некоторых классах $A H$-структур на касательном расслоении // Труды международной конференции, посвященной А. З. Петрову, 2000. С. 53-54.

[11] Zayatuev B. V. About some classes of almost Hermition structure on tangent bundele // Webs and Quasigroups. T. S. U. 2002. P. 103-106.

[12] Nakayama S. Conformal relations in almost Hermitian spaces // Tensor. 1962. V. 12. № 3. P. 278-289.

[13] Hervella L. M., Vidal E. Nouvelles geometries pseudo-kahleriennes $G_{1}$ et $G_{2} / /$ C. R. Acad. Sci. Paris. 1976. V. 283. P. 115-118.

[14] Gray A. The structure of nearly Kaehler manifolds // Ann. Math. 1976. V. 223. № 3. P. 233-248.

[15] Кириченко В.Ф.Дифференциальная геометрия К-пространств // Проблемы геометрии. Итоги науки и техники. Т. 8. М.: ВИНИТИ АН СССР, 1977. С. 139-161.

[16] Лихнерович А. Теория связностей в целом и группы голономии. М.: ИЛ, 1960.

[17] Kirichenko V.F. Geneneralized quasi-Kaehlerian manifolds and axioms of $C R$-submanifolds in generalized Hermitian geometry. II // Geom. Dedicata. 1994. V. 52. P. 53-85.

[18] Apte M. Sur certaines varietes hermetiques // C. R. Acad. Sci. Paris. 1954. V. 238. № 19. P. 1091-1093. 